\title{
New steps of robot-assisted radical prostatectomy using the extraperitoneal approach: a propensity-score matched comparison between extraperitoneal and transperitoneal approach in Japanese patients
}

Satoshi Kurokawa ${ }^{1,2^{*}}$ (D), Yukihiro Umemoto ${ }^{2}$, Kentaro Mizuno ${ }^{2}$, Atsushi Okada $^{2}$, Akihiro Nakane ${ }^{2}$, Hidenori Nishio ${ }^{2}$, Shuzo Hamamoto ${ }^{2}$, Ryosuke Ando ${ }^{2}$, Noriyasu Kawai ${ }^{2}$, Keiichi Tozawa ${ }^{2}$, Yutaro Hayashi ${ }^{2}$ and Takahiro Yasui $^{2}$

\begin{abstract}
Background: Robot-assisted radical prostatectomy (RARP) is commonly performed using the transperitoneal (TP) approach with six trocars over an 8-cm distance in the steep Trendelenburg position. In this study, we investigated the feasibility and the benefit of using the extraperitoneal (EP) approach with six trocars over a 4-cm distance in a flat or $5^{\circ}$ Trendelenburg position. We also introduced four new steps to the surgical procedure and compared the surgical results and complications between the EP and TP approach using propensity score matching.

Methods: Between August 2012 and August 2016, 200 consecutive patients without any physical restrictions underwent RARP with the EP approach in a less than $5^{\circ}$ Trendelenburg position, and 428 consecutive patients underwent RARP with the TP approach in a steep Trendelenburg position. Four new steps to RARP using the EP approach were developed: 1) arranging six trocars; 2) creating the EP space using laparoscopic forceps; 3 ) holding the separated prostate in the EP space outside the robotic view; and 4) preventing a postoperative inguinal hernia. Clinicopathological results and complications were compared between the EP and TP approaches using propensity score matching. Propensity scores were calculated for each patient using multivariate logistic regression based on the preoperative covariates.
\end{abstract}

Results: All 200 patients safely underwent RARP using the EP approach. The mean volume of estimated blood loss and duration of indwelling urethral catheter use were significantly lower with the EP approach than the TP approach (139.9 vs $184.9 \mathrm{~mL}, p=0.03$ and 5.6 vs 7.7 days, $p<0.01$, respectively). No significant differences in the positive surgical margin were observed. None of the patients developed an inguinal hernia postoperatively after we introduced this technique. (Continued on next page)

\footnotetext{
* Correspondence: sakun1974@hotmail.com

'Department of Urology, Nagoya Tokushukai General Hospital, 2-52,

Kouzouji-cho-kita, Kasugai 487-0016, Japan

${ }^{2}$ Department of Nephro-urology, Nagoya City University Graduate School of

Medical Sciences, 1, Kawasumi, Mizuho-cho, Mizuho-ku, Nagoya 467-8601,

Japan
} 
(Continued from previous page)

Conclusions: The EP approach to RARP was safely performed regardless of patient physique or contraindications to a steep Trendelenburg position. Our method, which involved using the EP approach to perform RARP, can decrease the amount of perioperative blood loss, the duration of indwelling urethral catheter use, and the incidence of postoperative inguinal hernia development.

Keywords: Robot-assisted radical prostatectomy, Extraperitoneal approach, Transperitoneal approach, Small physique, Trendelenburg position, Inguinal hernia, Propensity score

\section{Background}

Robot-assisted radical prostatectomy (RARP) has been used worldwide since it was introduced in $2000[1,2]$. The 4-armed da Vinci ${ }^{\oplus} \mathrm{S}$ surgical system (Intuitive Surgical, Sunnyvale, CA, USA), which is responsible for the widespread use of RARP, has 8-cm-wide arms, and the trocars should be spaced at intervals $>8 \mathrm{~cm}$ to prevent them from colliding with each other [3]. RARP is commonly performed using the transperitoneal (TP) approach because it offers enough arm distance, a larger working space, and familiar laparoscopic intraperitoneal landmarks [2]. The working space is relatively smaller when using the extraperitoneal (EP) approach. However, the procedure can be performed with little effect on patients with previous intraabdominal surgery or severe obesity [4], and it causes minimal intraabdominal complications [2, 5]. Furthermore, because the steep Trendelenburg position is not required, the EP approach is effective in patients with contraindications to this position [6, 7]. However, the EP approach has several limitations including a small working space and collision of the robotic arms with one another. Within a small operating cavity, the separated prostate impedes the operator's visibility, and performing the vesicourethral anastomosis becomes difficult. It is challenging to acquire enough space to keep the removed prostate out of the robotic view and avoid collision of the robotic arms in patients who are physically small in particular. The development of new methods is necessary to overcome these difficulties.

Herein, we introduce four new steps for the surgical procedure and investigate the feasibility of using the EP approach with six trocars over a 4-cm distance in a less than $5^{\circ}$ Trendelenburg position. We also compare the surgical results and complications of RARP using the EP and TP approaches.

\section{Methods}

The study protocol was approved by the Tokushukai Group Ethical Committee (approval number: TGE00700-016). Clinical data were gathered starting from the beginning of our experience with RARP. The database included preoperative, operative, and postoperative information.
From August 2012 to August 2016, we retrospectively reviewed the data of 200 consecutive patients who underwent RARP with the EP approach in a flat or $5^{\circ}$ Trendelenburg position and 428 consecutive patients who underwent RARP with the TP approach in a steep Trendelenburg position. Our surgical team began performing RARP using the TP approach in May 2011. Prior to that, they had experience performing $>600$ laparoscopic radical prostatectomies using TP and EP approaches [8-11]. After we managed 135 cases of RARP with the TP approach, we began performing RARP with the EP approach.

\section{Preoperative, operative, and postoperative data}

Preoperative clinical data pertaining to patient background and prostate cancer such as the prostate-specific antigen (PSA) value, Gleason score, and clinical T stage were collected. Patient background included age, physique (height, weight, and body mass index [BMI]), and medical history. Patients with glaucoma, severe valvular heart disease, or intracranial diseases (e.g., an unruptured cerebral aneurysm) were submitted a priori to RARP using the EP approach in advance to avoid complications from the Trendelenburg position. Operative characteristics including the total operative time, robot console time, vesicourethral anastomosis time, estimated volume of blood loss, weight of the removed prostate, performance of lymph-node dissection, and surgical complications were also studied. Pathological variables included the Gleason score, presence of extension, seminal vesicle invasion, and surgical margin status. Postoperative information including the duration of indwelling urethral catheters and presence of postoperative complications was collected. Functional outcome was assessed based on urinary continence 6 months postoperatively. Continence was defined as using no pads or one safety pad per day.

\section{Surgical procedure}

All the surgeries were conducted by the same surgical team using the da $\mathrm{Vinci}^{\oplus} \mathrm{S}$ surgical system. All surgeons had reached the plateau in the learning curve having had experience with more than 50 cases $[12,13]$. RARP 
using the TP approach was performed as described in previous articles $[14,15]$. RARP using the EP approach was performed as follows.

First, we determined the position of the six trocars whose distances were $4 \mathrm{~cm}$ apart when performing RARP using the EP approach (Fig. 1). Traditionally, trocars are placed 7-8 $\mathrm{cm}$ apart during RARP using the EP approach [16-18]. However, it was difficult to space the trocars $>7 \mathrm{~cm}$ apart in patients with small physiques. The decision to space the trocars $4 \mathrm{~cm}$ apart was based on a recommendation for performing robotic surgery in children [19].

Second, we created the EP space using a balloon dilator, finger assistance, and laparoscopic forceps. Furthermore, we developed a new technique for placing the trocar in a small space. A 4-cm transverse infraumbilical incision was made through the anterior rectus sheath. We intended to dissect the EP space digitally at the position of the trocars of robotic arm no. 1 or no. 2. We inserted a balloon dilator (Pajunk ${ }^{\circ}$ balloon systems, Pajunk, Germany) and created the EP space using the laparoscopic view. If the EP space was insufficient to insert all six trocars, it was expanded using laparoscopic forceps through the trocar of robotic arm no. 1 or no. 2 as a working port. When placing a trocar in a small adhesive space, there is a risk for the trocar to become lost in the adhesion tissue. Therefore, we used a laparoscopic dissector with a pointed tip to penetrate the abdominal wall from the small space of the EP cavity to the outside of the body (Fig. 2). The shaft of the laparoscopy forceps was placed along the inner cylinder, and it was possible to extracorporeally guide the outer tube of the 5-mm trocar. When attempting to guide the $12-\mathrm{mm}$ trocar, the tract of the trocar was gradually dilated after the $5-\mathrm{mm}$ trocar was inserted, followed by the insertion of the $8-\mathrm{mm}$ and 12-mm trocars.

Third, we prepared to hold the freed prostate in the right abdominal EP space outside of robotic view. A 14-gauge intravenous indwelling needle was penetrated into the EP space from outside of the abdominal wall between the no. 1 and no. 3 robotic trocars (Fig. 1). After inserting a 14-gauge needle, the plastic cannula (outer cylinder) was left and the inner time (metal needle) removed. The plastic cannula was closed with the lid of the three-way stopcock to prevent carbon dioxide gas from leaking. After the prostate was dissected from the bladder neck in an antegrade manner, the separated prostate was stored in a pouch. Then, the thread of the pouch was pulled outside of the body through a 14-gauge plastic cannula (Fig. 3). The prostate was held on the right side of the abdomen outside of robotic view. After using the Rocco suture method, vesicourethral anastomosis was performed using the single-knot method with Lapra-Ty clips [9, 20].

Fourth, to prevent an indirect inguinal hernia postoperatively, we dissected and peeled the peritoneum from the spermatic cord at the inner inguinal ring. This technique was originally performed during retropubic radical prostatectomy [21], and it was introduced in RARP using the EP approach for all patients after the twenty-first patient. The cord-like structures of the cremaster muscle and fibrous tissue were transected around the spermatic cord, and the testicular vessels and vas deference were separated (Fig. 4).

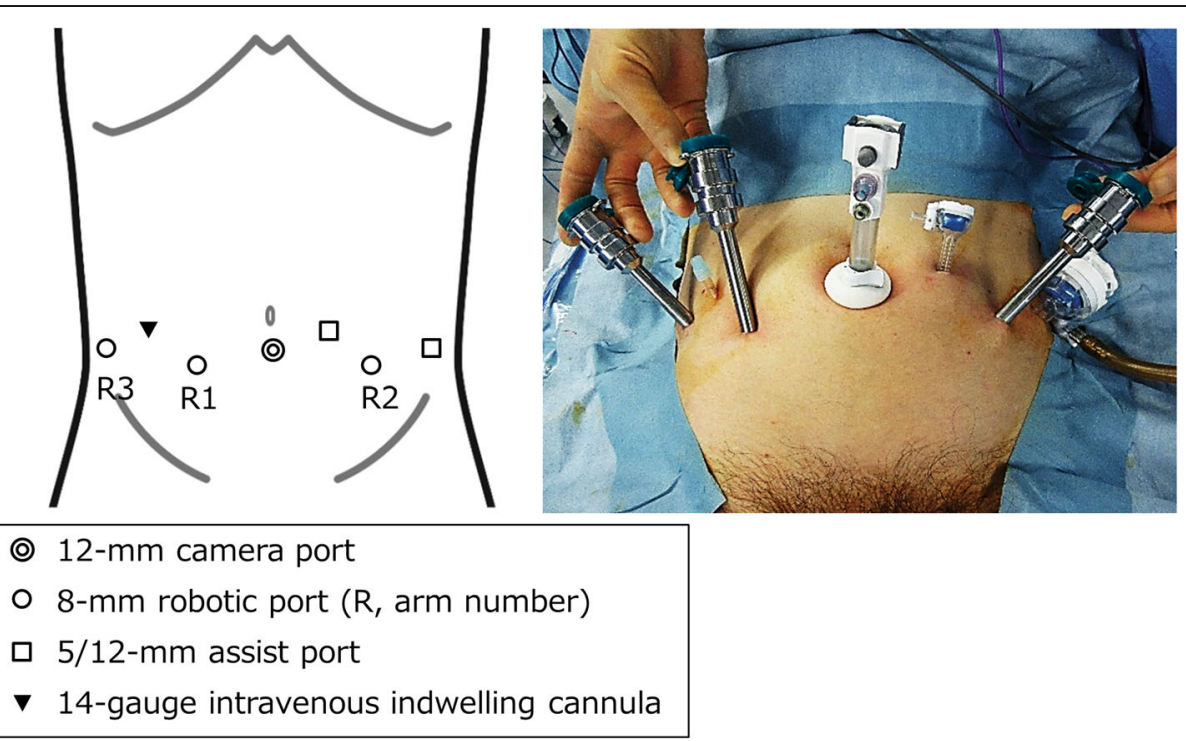

Fig. 1 Placement of ports in the extraperitoneal approach to robot-assisted radical prostatectomy. In addition to six trocars, we place a 14-gauge intravenous indwelling cannula in the right lower abdominal region 


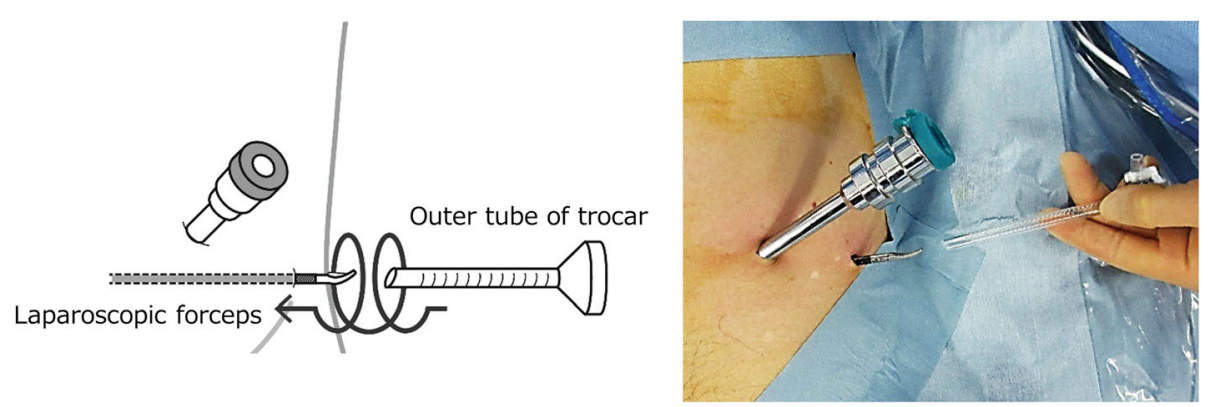

Fig. 2 Method of placing a trocar in a small space (left side, assist port). We use laparoscopic forceps (a curved dissector) to penetrate from the small space of the extraperitoneal cavity to outside the body. Forceps are used instead of the inner cylinder of the trocar and only the outer tube of the trocar guides insertion of the forceps

Finally, the prostate was removed, and a 10-French closed drain was indwelled. The drain was removed on postoperative day 2 , and on postoperative days $4-7$, the urethral catheter was removed after confirming that there was no leakage using cystourethrography.

In selected patients whose risk of lymph node metastases was $>10 \%$ according to the preoperative nomogram for Japanese patients or who were classified into the high-risk group according to the D'Amico criteria, pelvic lymph-node dissection was performed after the fourth step of preventing an inguinal hernia $[22,23]$.

\section{Propensity score matching and statistical analysis}

Propensity scores were calculated for each patient using multivariate logistic regression analysis based on the preoperative covariates. The covariates used to compare the EP and TP groups were: follow-up duration, age, height, weight, BMI, prostate-specific antigen value, biopsy Gleason score, and clinical T stage. The covariates of the chronological comparison in the EP group excluded follow-up duration. Subsequently, balance of matching was assessed using statistical comparison. In propensityscore matched groups of the EP and TP approach, we analyzed operative results and postoperative outcomes.
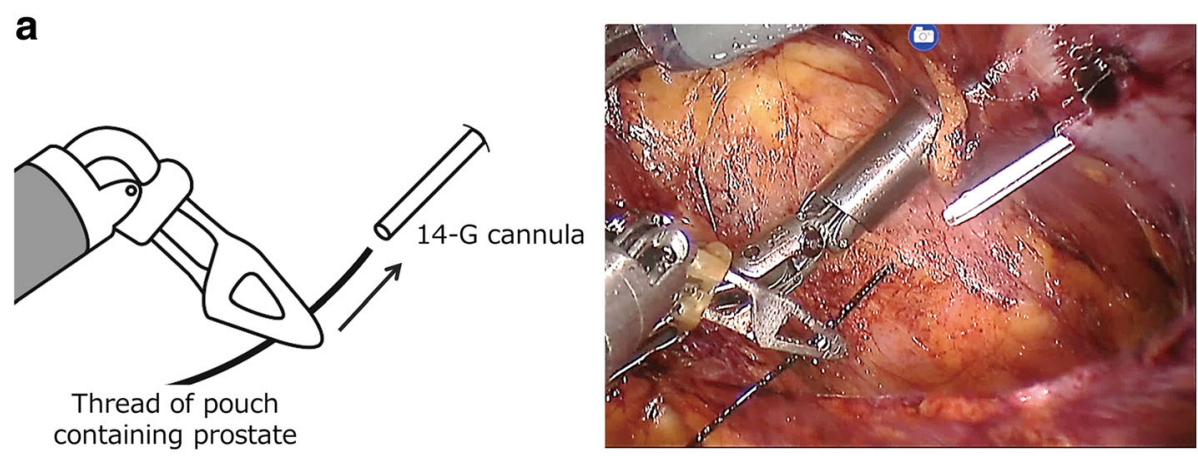

b
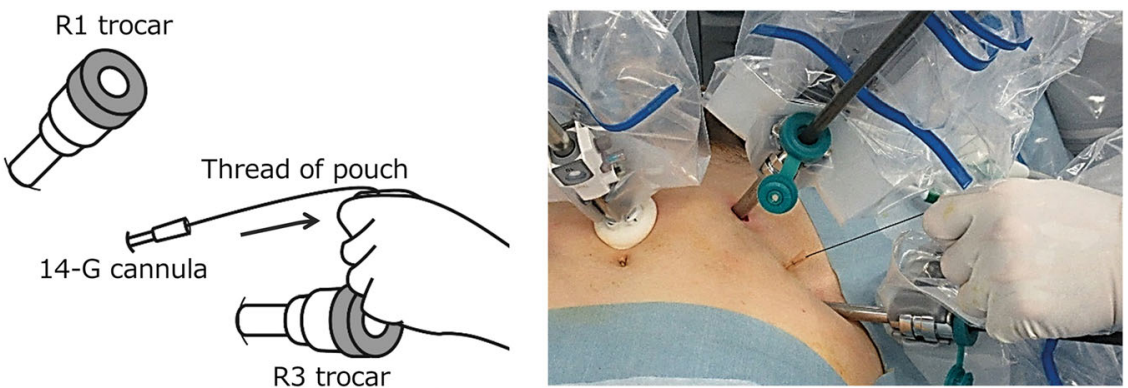

Fig. 3 The prostate is placed in a pouch and retracted to the lower right abdomen. The thread of the pouch containing the prostate is pulled out of the body through the 14-gauge intravenous indwelling cannula (a-b) G, gauge 

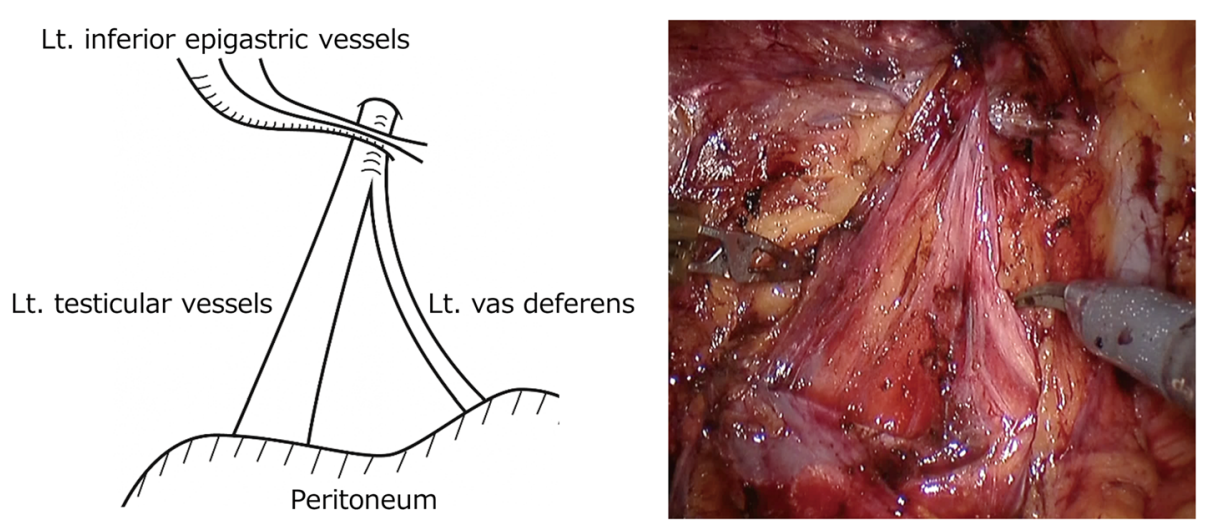

Fig. 4 Technique to prevent postoperative inguinal hernia (Lt side). The peritoneum is dissected and peeled from the spermatic cord on the cranial side of the inner inguinal ring. Cord-like structures between the spermatic cord and peritoneum are transected, and the spermatic cord is separated into the testicular vessels and vas deferens $L t$, left

In the two propensity-score matched chronological subgroups in the EP group, operative and postoperative outcomes were also compared. The comparisons were evaluated using an $x^{2}$-test for qualitative variables and the Mann-Whitney $\mathrm{U}$ test for quantitative variables. A two-tailed $p$-value $<0.05$ was considered significant. Statistical analysis was performed with the JMP $13.0^{\circ}$ software (SAS Institute Corp., Cary, NC, USA).

\section{Results}

Patients in the EP and TP group were balanced using propensity score matching (Table 1 ). The patient with the smallest physique in the EP group was $143.6 \mathrm{~cm}$ tall and weighed $34.8 \mathrm{~kg}$; he underwent RARP using the EP approach safely. Patient backgrounds such as follow-up duration, age, height, weight, and BMI were not significantly different between the groups (Table 1). Preoperative clinical characteristics such as the mean PSA level, biopsy Gleason score, clinical stage, and previous abdominal surgery, were also not significantly different between the groups (Table 1).

Table 2 shows the operative and postoperative results, as well as complications in both groups. The mean operative time was significantly longer in the EP group than in the TP group ( 254.5 vs $225.8 \mathrm{~min}, p<0.01)$. The mean robot console time and vesicourethral anastomosis time were not significantly different between the EP and TP group (180.5 vs $175.4 \mathrm{~min}, p=0.07$; and 30.3 vs

Table 1 Patients' preoperative clinical characteristics by surgical procedures

\begin{tabular}{|c|c|c|c|}
\hline Variables & EP approach & TP approach & $p$-Value \\
\hline Patients (n) & 190 & 190 & \\
\hline Follow-up (months) & $27.3 \pm 13.8(4-52)$ & $27.8 \pm 154.3(4-52)$ & 0.78 \\
\hline Age (years) & $69.4 \pm 6.0(51-82)$ & $69.3 \pm 4.7(51-79)$ & 0.70 \\
\hline Height (cm) & $165.2 \pm 6.1(143.6-186.0)$ & $165.4 \pm 5.3(152.5-183.2)$ & 0.70 \\
\hline Weight (kg) & $63.7 \pm 9.3(34.8-103.0)$ & $63.6 \pm 9.4(38.0-122.0)$ & 0.89 \\
\hline BMI $\left(\mathrm{kg} / \mathrm{m}^{2}\right)$ & $23.3 \pm 3.0(16.9-34.5)$ & $23.2 \pm 2.9(15.8-36.3)$ & 0.73 \\
\hline PSA (ng/mL) & $10.7 \pm 11.6(2.2-84.4)$ & $10.8 \pm 10.4(2.3-73.1)$ & 0.24 \\
\hline Biopsy Gleason score (\%) & & & 0.12 \\
\hline $2-6$ & $36(19.0)$ & $44(23.2)$ & \\
\hline 7 & $100(52.6)$ & $80(42.1)$ & \\
\hline $8-10$ & $54(28.4)$ & $66(34.7)$ & \\
\hline Clinical stage (\%) & & & 0.15 \\
\hline $\mathrm{T} 1$ & $41(21.6)$ & $49(25.8)$ & \\
\hline $\mathrm{T} 2$ & $142(74.7)$ & $126(66.3)$ & \\
\hline T3 & $7(3.7)$ & $15(7.9)$ & \\
\hline Previous abdominal surgery (\%) & $62(32.3)$ & $65(35.4)$ & 0.91 \\
\hline
\end{tabular}

EP extraperitoneal, $T P$ transperitoneal, $B M I$ body mass index, $P S A$ prostate-specific antigen 
Table 2 Comparison of operative and postoperative results, and complications between the extraperitoneal and transperitoneal approaches

\begin{tabular}{|c|c|c|c|}
\hline Variables & EP approach & TP approach & $p$-Value \\
\hline Patients (n) & 190 & 190 & \\
\hline Operative time (min) & $254.5 \pm 42.5(144-464)$ & $225.8 \pm 44.0(131-410)$ & $<0.01$ \\
\hline Robot console time (min) & $180.5 \pm 31.7(98-304)$ & $175.4 \pm 40.9(98-351)$ & 0.07 \\
\hline Anastomosis time (min) & $30.3 \pm 11.7(7-73)$ & $28.0 \pm 10.0(8-66)$ & 0.09 \\
\hline Blood loss (mL) & $139.9 \pm 118.7(5-800)$ & $184.9 \pm 195.8(0-1485)$ & 0.03 \\
\hline Prostate weight (g) & $46.0 \pm 13.1(22-96)$ & $44.8 \pm 16.4(8-132)$ & 0.13 \\
\hline Indwelling urethral catheter (days) & $5.6 \pm 1.7(4-16)$ & $7.7 \pm 3.7(4-33)$ & $<0.01$ \\
\hline Pathological Gleason score (\%) & & & 0.02 \\
\hline $2-6$ & $24(12.6)$ & $21(11.1)$ & \\
\hline 7 & $118(62.1)$ & $142(74.7)$ & \\
\hline $8-10$ & $54(28.4)$ & $27(14.2)$ & \\
\hline Pathological stage (\%) & & & $<0.01$ \\
\hline pT2 & $121(63.7)$ & $153(80.5)$ & \\
\hline pT3 & $69(36.3)$ & $37(19.5)$ & \\
\hline \multicolumn{4}{|l|}{ Positive surgical margin (\%) } \\
\hline pT2 & $13(10.7)$ & 30 (19.6) & 0.09 \\
\hline pT3 & $37(53.6)$ & $21(56.8)$ & 0.76 \\
\hline Lymphadenectomy (\%) & $63(33.2)$ & $71(37.4)$ & 0.22 \\
\hline Continence (\%) & $173(91.1)$ & $170(89.5)$ & 0.60 \\
\hline \multicolumn{4}{|l|}{ Complications (\%) } \\
\hline Anastomosis stenosis & $2(1.1)$ & $5(2.6)$ & 0.20 \\
\hline Blood transfusion & $0(0.0)$ & $1(0.5)$ & 0.32 \\
\hline Colorectal injury & $0(0.0)$ & $2(1.1)$ & 0.16 \\
\hline Conversion to open surgery & $0(0.0)$ & $1(0.5)$ & 0.32 \\
\hline lleus & $0(0.0)$ & $2(1.1)$ & 0.16 \\
\hline Indirect inguinal hernia & $2(1.1)$ & $14(7.4)$ & $<0.01$ \\
\hline Symptomatic lymphocele & $0(0.0)$ & $1(0.5)$ & 0.32 \\
\hline
\end{tabular}

EP extraperitoneal, $T P$ transperitoneal

$28.0 \mathrm{~min}, p=0.09$, respectively). The mean volume of estimated blood loss and duration of indwelling urethral catheters were significantly lower in the EP group than in the TP group (139.9 vs $184.9 \mathrm{~mL}, p<0.01$; and 5.6 vs 7.7 days, $\mathrm{p}<0.01$, respectively). There were no significant differences between the groups in terms of the positive surgical margin, lymph-node dissection rate, and urinary continence. The rate of postoperative inguinal hernia was significantly lower in the EP group than in the TP group $(1.1$ vs $7.4 \%, \mathrm{p}<0.01)$. There were no cases of conversion to open surgery in the EP group.

Table 3 compares the surgical and pathological results of the 200 patients in the EP group subdivided into two chronological groups of 100 patients. The mean operative time, robot console time, and vesicourethral anastomosis time were significantly shorter in the latter group (257.9 vs $247.2 \mathrm{~min}, \mathrm{p}<0.01 ; 184.5$ vs $174.1 \mathrm{~min}$, $\mathrm{p}<0.01$; and 33.4 vs $24.2 \mathrm{~min}, \mathrm{p}<0.01$, respectively). None of the patients developed an indirect inguinal hernia postoperatively after our method was introduced.

\section{Discussion}

Our method of RARP using the EP approach can be safely implemented, even in patients with small physiques. RARP using the EP approach has the benefit of reducing the amount of blood loss and shortening the duration of indwelling urethral catheter use compared with RARP using the TP approach.

We divided the surgical procedure into four steps. First, we arranged six trocars spaced $4 \mathrm{~cm}$ apart. In all 200 patients in the EP group, it was possible to perform surgery without revising the trocar placement. This result suggests that it is possible to perform RARP with the EP approach using six trocars spaced $4 \mathrm{~cm}$ apart. 
Table 3 Comparison of outcomes of the extraperitoneal approach according to the extent of surgical experience

\begin{tabular}{|c|c|c|c|}
\hline \multirow[b]{2}{*}{ Variables } & \multicolumn{2}{|l|}{ EP approach } & \multirow[b]{2}{*}{$p$-Value } \\
\hline & $1-100$ & $101-200$ & \\
\hline Patients (n) & 82 & 82 & \\
\hline Operative time (min) & $258.6 \pm 40.5$ & $244.1 \pm 43.2$ & $<0.01$ \\
\hline Robot console time (min) & $183.5 \pm 30.9$ & $169.9 \pm 26.2$ & $<0.01$ \\
\hline Anastomosis time (min) & $33.1 \pm 10.5$ & $22.9 \pm 7.7$ & $<0.01$ \\
\hline Blood loss (mL) & $157.3 \pm 116.6$ & $104.8 \pm 95.7$ & $<0.01$ \\
\hline Prostate weight (g) & $46.3 \pm 11.6$ & $46.7 \pm 14.4$ & 0.93 \\
\hline Indwelling urethral catheter (days) & $5.2 \pm 1.0$ & $5.6 \pm 1.6$ & 0.20 \\
\hline Pathological Gleason score (\%) & & & 0.04 \\
\hline $2-6$ & $14(17.1)$ & $4(4.9)$ & \\
\hline 7 & $48(58.5)$ & $55(67.1)$ & \\
\hline $8-10$ & $20(24.4)$ & $23(28.0)$ & \\
\hline Pathological stage (\%) & & & 0.34 \\
\hline pT2 & $53(64.6)$ & $47(57.3)$ & \\
\hline pT3 & $29(35.4)$ & $35(42.7)$ & \\
\hline \multicolumn{4}{|l|}{ Positive srgical margin (\%) } \\
\hline pT2 & $6(11.3)$ & $2(4.3)$ & 0.15 \\
\hline pT3 & $20(69.0)$ & $12(34.3)$ & $<0.01$ \\
\hline Lymphadenectomy (\%) & $26(31.7)$ & $31(37.8)$ & 0.25 \\
\hline Continence (\%) & $73(89.0)$ & 76 (92.7) & 0.42 \\
\hline \multicolumn{4}{|l|}{ Complications (\%) } \\
\hline Indirect inguinal hernia & $2(2.4)$ & $0(0.0)$ & 0.16 \\
\hline Anastomosis stenosis & $1(1.2)$ & $1(1.2)$ & 1.00 \\
\hline
\end{tabular}

Second, we created the EP space and guided the trocar with a new technique. Normally, the trocar is placed using finger assistance or laparoscopy to confirm the tip of the trocar so that it does not damage the surrounding structures [24]. However, when the location of trocar placement cannot be sufficiently exposed because of adhesions, it is difficult to appropriately confirm the tip of the trocar. Therefore, we used the laparoscopic dissector to penetrate from the EP space to outside of the body, to avoid the adhesive site, and to guide the trocar. This technique is a useful way to place the trocar into a narrow space, and it can be used during robotic surgery as well as any laparoscopic surgery.

Third, we developed a new technique to hold the separated prostate in the EP space. A 14-gauge cannula can serve as a tract for the thread of the pouch. During robotic or laparoscopic pyeloplasty, a 14-gauge cannula is used as a tract for the insertion of a ureteral stent [25, 26]. Thus, using an intravenous indwelling needle as a small tract through the abdominal wall can be helpful during minimally invasive surgery.
Fourth, the technique of preventing a postoperative inguinal hernia was simple and effective. This technique was originally performed during retropubic radical prostatectomy [21]. Before we introduced this technique, a postoperative inguinal hernia developed in $2(10 \%)$ patients. However, none of the patients developed postoperative inguinal hernia after introducing this technique. The average follow-up duration in our study was over 27 months. The average interval between prostatectomy and postoperative inguinal hernia diagnosis was reported to be 10.6 months [21]. Thus, the follow-up duration we used in our study was sufficient to determine the effect of this technique.

Urethral anastomosis stenosis was observed in $1.1 \%$ of patients in the EP group and $2.6 \%$ of patients in the TP group. The incidence of strictures of the vesico-urethral anastomosis after radical prostatectomy has been reported to range from 0.5 to $32 \%$, with most occurring within 5 months of radical prostatectomy [27-29]. Thus, the rate of anastomosis stenosis in our study was lower than those in the literature and the follow-up duration of our study was sufficient to evaluate the rate of anastomosis stenosis after RARP.

Symptomatic lymphocele occurred in $0 \%$ of patients in the EP group and $0.5 \%$ of patients in the TP group. We removed drains on postoperative day 2 after confirming a decrease in the drain fluid. The duration of pelvic drainage has been reported to influence the rate of symptomatic lymphocele [30]. The group whose drains were removed on postoperative day 1 exhibited higher rates of symptomatic lymphocele than patients whose drains were removed on postoperative day 7 and patients without drainage [30]. In general, lymphatic fluid cannot be absorbed by the peritoneal surface in the EP approach. Accordingly, the rate of lymphocele in the EP approach is greater than that in the TP approach. During the postoperative course of our study, we removed the pelvic drain after the amount of drain fluid decreased. Thus, the rate of lymphocele was low compared with that in the literature [31].

Our four new steps were demonstrated to be successful for performing RARP using the EP approach safely. The positive surgical margin and the time of operation, robot console, and vesicourethral anastomosis were compatible to those in the literature [32]. In particular, the amount of the blood loss and the incidence of the postoperative complications, such as inguinal hernia, anastomosis stenosis, and lymphocele, were low compared with those in the literature [31, 32].

The robot console time and vesicourethral anastomosis time of the EP approach became shorter after 100 cases, and they became shorter than those of the TP approach. However, the total operative time of the EP approach was still longer after 100 cases than that of the TP approach. 
There is a possibility that extra time was needed to create the EP space. As the operative space is relatively smaller in the EP approach, bleeding should be carefully controlled to maintain the robotic view. Vesicourethral anastomosis was performed after completing hemostasis. The values of blood loss and duration of indwelling urethral catheter use were significantly lower from the beginning of the EP approach than those of the TP approach. We first started RARP using the TP approach, and we overcame the learning curve of $50-100$ cases before starting to perform the EP approach [12, 13]. However, 100 cases of the EP approach were needed to overcome the learning curve of EP approach.

We acknowledge that our study had strengths and weaknesses. One strength of our study is that we created a propensity-score matched comparison, which had a balanced covariate preoperative factor profiling, reducing the possibility of cofounding. Although our study was retrospectively designed, the propensity-score matched comparison might reduce selection bias. There were a few additional limitations to our study. First, the sample size might be relatively small for a study of this nature. Second, the model for propensity score matching may not include potentially relevant factors that are simply unknown at this time. Third, erectile data evaluation was not performed. Fourth, the operation was performed by the same surgical team but not by a single surgeon, which might have had an impact on the outcomes of the operation and the postoperative course.

\section{Conclusions}

RARP with the EP approach using four new steps was safely performed regardless of patient physique and medical history. Our method of RARP with the EP approach can reduce the amount of perioperative blood loss, the duration of indwelling urethral catheters, and the incidence of postoperative inguinal hernia development. In addition to RARP using the TP approach, the EP approach is useful and it should be learned and mastered for all types of patients regardless of their medical history, if possible.

\section{Abbreviations}

BMI: Body mass index; DVC: Deep vein complex; EP: Extraperitoneal; PSA: Prostate-specific antigen; RARP: Robot-assisted radical prostatectomy; TP: Transperitoneal

\section{Acknowledgements}

None

\section{Funding}

None.

\section{Availability of data and materials}

The datasets generated and/or analyzed during the current study are not publicly available due to ethical restrictions but are available from the corresponding author upon reasonable request.

\section{Authors' contributions}

SK, YU, KM, AO, AN, HN, SH, RA, NK, KT, and TY were members of our surgical team, and SK, YU, KT, and TY were surgeons with experience in over 50 cases, which is the plateau of the learning curve. SK contributed to the conception, design, analyses, and interpretation of the data. In addition, SK also drafted the manuscript, and agreed to be accountable for the accuracy and all other aspects of the work. SK and SH contributed to the data entry and management. YH and TY did the supervision job throughout this study. All authors read and approved the final manuscript.

\section{Ethics approval and consent to participate}

The study protocol was approved by the Tokushukai Group Ethical Committee (approval number: TGE00700-016). A waiver of consent was obtained as part of the ethical committee approval; therefore, patients included in this study were not required to provide consent.

\section{Consent for publication}

Not applicable

\section{Competing interests}

The authors declare that they have no competing interests.

\section{Publisher's Note}

Springer Nature remains neutral with regard to jurisdictional claims in published maps and institutional affiliations.

Received: 31 January 2017 Accepted: 13 November 2017

Published online: 21 November 2017

\section{References}

1. Abbou CC, Hoznek A, Salomon L, Lobontiu A, Saint F, Cicco A, et al. Remote laparoscopic radical prostatectomy carried out with a robot. Report of a case. Prog Urol. 2000;10:520-3.

2. Li-Ming S, Scott MG, Joseph AS Jr. Laparoscopic and robotic-assisted laparoscopic radical prostatectomy and pelvic lymphadenectomy. In: Wein AJ, Kavoussi LR, Partin AW, Peters CA, editors. Campbell-Walsh urology. 11th ed. Philadelphia: Elsevier; 2016. p. 2663-8.

3. Joseph AS Jr. Robot-assisted laparoscopic prostatectomy. In: Joseph Jr AS, Stuart SH, Edward JM, Glenn MP, editors. Hinman's atlas of urologic surgery. 3rd ed. Philadelphia: Elsevier; 2012. p. 435-6.

4. Agrawal V, Feng C, Joseph J. Outcomes of extraperitoneal robot-assisted radical prostatectomy in the morbidly obese: a propensity score-matched study. J Endourol. 2015;29:677-82.

5. Akand M, Erdogru T, Avci E, Ates M. Transperitoneal versus extraperitoneal robot-assisted laparoscopic radical prostatectomy: a prospective single surgeon randomized comparative study. Int J Urol. 2015;22:916-21.

6. Ventura LM, Golubev I, Lee W, Nose I, Parel JM, Feuer WJ, et al. Head-down posture induces PERG alterations in early glaucoma. J Glaucoma. 2013;22:255-64.

7. Haas S, Haese A, Goetz AE, Kubitz JC. Haemodynamics and cardiac function during robotic-assisted laparoscopic prostatectomy in steep Trendelenburg position. Int J Med Robot. 2011;7:408-13.

8. Nakane A, Akita H, Okamura T, Nagata D, Kojima Y, Akita H, et al. Feasibility of a novel extraperitoneal two-port laparoendoscopic approach for radical prostatectomy: an initial study. Int J Urol. 2013;20:729-33.

9. Naiki T, Kawai N, Okamura T, Nagata D, Kojima Y, Akita H, et al. Neoadjuvant hormonal therapy is a feasible option in laparoscopic radical prostatectomy. BMC Urol. 2012;12:36.

10. Yasui T, Itoh Y, Maruyama T, Akita H, Hashimoto Y, Tozawa K, et al. The single-knot method with Lapra-Ty clips is useful for training surgeons in vesicourethral anastomosis during laparoscopic radical prostatectomy. Int Urol Nephrol. 2009;41:281-5.

11. Tozawa K, Hashimoto Y, Yasui T, Itoh Y, Nagata D, Akita H, et al. Evaluation of operative complications related to laparoscopic radical prostatectomy. Int J Urol. 2008;15:222-5.

12. Hashimoto T, Yoshioka K, Gondo T, Kamoda N, Satake N, Ozu C, et al. Learning curve and perioperative outcomes of robot-assisted radical prostatectomy in 200 initial Japanese cases by a single surgeon. J Endourol. 2013;27:1218-23.

13. Doumerc N, Yuen C, Savdie R, Rahman MB, Rasiah KK, Pe Benito R, et al. Should experienced open prostatic surgeons convert to robotic surgery? The real learning curve for one surgeon over 3 years. BJU Int. 2010;106:378-84. 
14. Yasui T, Tozawa K, Okada A, Kurokawa S, Kubota H, Mizuno K, et al. Outcomes of robot-assisted laparoscopic prostatectomy with a posterior approach to the seminal vesicle in 300 patients. Int Sch Res Notices. 2014;2014:565737.

15. Yasui T, Tozawa K, Kurokawa S, Okada A, Mizuno K, Umemoto Y, et al. Impact of prostate weight on perioperative outcomes of robot-assisted laparoscopic prostatectomy with a posterior approach to the seminal vesicle. BMC Urol. 2014;14:6.

16. Lee JY, Diaz RR, Cho KS, Yu HS, Chung JS, Ham WS, et al. Lymphocele after extraperitoneal robot-assisted radical prostatectomy: a propensity scorematching study. Int J Urol. 2013;20:1169-76.

17. Dogra PN, Saini AK, Singh P, Bora G, Nayak B. Extraperitoneal robot-assisted laparoscopic radical prostatectomy: initial experience. Urol Ann. 2014;6:130-4

18. Joseph JV, Rosenbaum R, Madeb R, Erturk E, Patel HR. Robotic extraperitoneal radical prostatectomy: an alternative approach. J Urol. 2006;175:945-50.

19. Casale P. Principles of laparoscopic and robotic surgery in children. In: Wein AJ, Kavoussi LR, Partin AW, Peters CA, editors. Campbell-Walsh urology. 11th ed. Philadelphia: Elsevier; 2016. p. 2969-74.

20. Van Velthoven RF, Ahlering TE, Peltier A, Skarecky DW, Clayman RV. Technique for laparoscopic running urethrovesical anastomosis: the single knot method. Urology. 2003;61:699-702.

21. Taguchi K, Yasui T, Kubota H, Fukuta K, Kobayashi D, Naruyama H, et al. Simple method of preventing postoperative inguinal hernia after radical retropubic prostatectomy. Urology. 2010;76:1083-7.

22. Naito S, Kuroiwa K, Kinukawa N, Goto K, Koga H, Ogawa O, et al. Validation of Partin tables and development of a preoperative nomogram for Japanese patients with clinically localized prostate cancer using 2005 International Society of Urological Pathology consensus on Gleason grading: data from the Clinicopathological research Group for Localized Prostate Cancer. J Urol. 2008;180:904-9.

23. D'Amico AV, Whittington R, Malkowicz SB, Schultz D, Blank K, Broderick GA, et al. Biochemical outcome after radical prostatectomy, external beam radiation therapy, or interstitial radiation therapy for clinically localized prostate cancer. JAMA. 1998:280:969-74.

24. Asimakopoulos AD, Mugnier C, Hoepffner JL, Lopez L, Rey D, Gaston R, et al. Laparoscopic treatment of benign prostatic hyperplasia (BPH): overview of the current techniques. BJU Int. 2011;107:1168-82.

25. Mizuno K, Kojima Y, Kurokawa S, Kamisawa H, Nishio H, Moritoki Y, et al. Robot-assisted laparoscopic pyeloplasty for ureteropelvic junction obstruction: comparison between pediatric and adult patients-Japanese series. J Robot Surg. 2016; doi: 10.1007/s11701-016-0633-5.

26. Kojima Y, Umemoto Y, Mizuno K, Tozawa K, Kohri K, Hayashi Y. Comparison of laparoscopic pyeloplasty for ureteropelvic junction obstruction in adults and children: lessons learned. J Urol. 2011;185:1461-7.

27. Song J, Eswara J, Brandes SB. Postprostatectomy anastomosis stenosis: a systematic review. Urology. 2015;86:211-8.

28. Pfalzgraf D, Siegel FP, Kriegmair MC, Wagener N. Bladder neck contracture after radical prostatectomy: what is the reality of care? J Endourol. 2017;31:50-6.

29. Park R, Martin S, Goldberg JD, Lepor H. Anastomotic strictures following radical prostatectomy: insights into incidence, effectiveness of intervention, effect on continence, and factors predisposing to occurrence. Urology. 2001;57:742-8.

30. Danuser H, Di Pierro GB, Stucki P, Mattei A. Extended pelvic lymphadenectomy and various radical prostatectomy techniques: is pelvic drainage necessary? BJU Int. 2013;111:963-9.

31. Chung JS, Kim WT, Ham WS, Yu HS, Chae Y, Chung SH, et al. Comparison of oncological results, functional outcomes, and complications for transperitoneal versus extraperitoneal robot-assisted radical prostatectomy: a single surgeon's experience. J Endourol. 2011;25:787-92.

32. Atug F, Castle EP, Woods M, Srivastav SK, Thomas R, Davis R. Transperitoneal versus extraperitoneal robotic-assisted radical prostatectomy: is one better than the other? Urology. 2006;68:1077-81.

\section{Submit your next manuscript to BioMed Central and we will help you at every step:}

- We accept pre-submission inquiries

- Our selector tool helps you to find the most relevant journal

- We provide round the clock customer support

- Convenient online submission

- Thorough peer review

- Inclusion in PubMed and all major indexing services

- Maximum visibility for your research

Submit your manuscript at www.biomedcentral.com/submit
Biomed Central 